\title{
Crystal plasticity-based creep model for solution-strengthened nickel-based alloys
}

\author{
Pritam Chakraborty* \\ Department of Aerospace Engineering, \\ IIT Kanpur, \\ Kanpur, India \\ Email: cpritam@iitk.ac.in
}

\section{Wen Jiang}

Idaho National Laboratory, Idaho Falls, Idaho, USA

Email: wen.jiang@inl.gov

*Corresponding author

\begin{abstract}
Nickel-based alloys are widely used in high temperature applications due to their favourable properties at extreme conditions. However, due to their high cost, efforts are being relentlessly made to extend the useful life of the components made from these alloys. Such life extension requires reliable constitutive models with detailed quantitative understanding of the factors contributing to property variations. Micromechanical analysis along with multi-scale methods can be a key enabler in developing the required high fidelity models. Particularly for properties such as creep that require long term prediction, accelerated tests with empirical models may prove insufficient. Thus, in the present work, a crystal plasticity-based creep model has been developed for solution strengthened nickel-based alloys. Through this model, the effect of microstructural variations in grain orientation, size, etc. on the secondary creep strain rate can be captured. The performance of the model is evaluated against creep data of alloy 617 .
\end{abstract}

Keywords: crystal plasticity; creep; finite element method; FEM; alloy 617.

Reference to this paper should be made as follows: Chakraborty, P. and Jiang, W. (2019) 'Crystal plasticity-based creep model for solution-strengthened nickel-based alloys', Int. J. Materials and Structural Integrity, Vol. 13, Nos. 1/2/3, pp.144-159.

Biographical notes: Pritam Chakraborty is an Assistant Professor in the Department of Aerospace Engineering at Indian Institute of Technology Kanpur. He received his $\mathrm{PhD}$ in Mechanical Engineering from The Ohio State University, USA following which he worked as a Staff Scientist in the Modelling and Simulation Department at Idaho National Laboratory, USA, before joining his present role. His research interests include computational mechanics and multi scale modelling of plasticity, fatigue, fracture and creep.

Wen Jiang is currently a computational Scientist in the Fuel Modelling and Simulation Department at Idaho National Laboratory (INL). He received his $\mathrm{PhD}$ in Mechanical Engineering and Materials Science from Duke University in 2015. After PhD, he joined INL as a Postdoctoral Researcher and worked in 


\begin{abstract}
the area of computational mechanics. He has extensive experience in computational methods and materials modelling. At INL, he is team member of MARMOT and BISON research group and develops mechanics capabilities in INL's MOOSE framework.
\end{abstract}

This paper is a revised and expanded version of a paper entitled 'Crystal plasticity based creep model for Ni-superalloys at polycrystalline length scale' presented at INCAM 2017, India, 5-7 July 2017.

\title{
1 Introduction
}

Solution strengthened nickel-based alloys have good stability, corrosion, oxidation and creep resistance, and, strength retention at elevated temperatures. Thus, these alloys are widely used in high temperature applications. For example, alloy 617 is expected to be used in high temperature ducts and heat exchangers in very high temperature nuclear reactors (Chomette et al., 2010). Since these alloys and the applications in which they are used typically involve high cost, ensuring the long term integrity at the elevated conditions involves a design as well as a material challenge. One such challenge is to ascertain the integrity of the components under long-term creep deformation. Over the years, a plethora of uniaxial creep experiments have been performed on these alloys and empirical models developed to address the same (Swindeman and Swindeman, 2008). Though these models can replicate the macroscopic behaviour, their applicability is limited to the particular heat and may be deficient for the same alloy composition but processed differently. This drawback can be addressed through numerical modelling of plasticity and damage processes (Giglio et al., 2013). Particularly for creep deformation, micromechanistic models and scale bridging can be employed to obtain engineering scale models with microstructure dependent parameters. Furthermore, micromechanistic models can provide a detailed understanding of creep deformation under multi-axial loading, and, stress and temperature gradients. The models can also provide mechanistic understanding of creep deformation in the welds of these alloys, with regions of heat affected and fusion zone.

The stress and temperature ranges expected on nickel-based alloy components that are to be used in very high temperature nuclear reactors and ultra supercritical steam turbines, are approximately $30-80 \mathrm{MPa}$ and $700-900^{\circ} \mathrm{C}$, respectively. The creep mechanisms within this window are dislocation climb and climb assisted glide (Lillo and Wright, 2015). For both of these mechanisms, vacancy diffusion in the bulk and at dislocation core, and, resolved shear stresses, are the driver. While the bulk vacancy diffusion processes can be treated as isotropic at single crystal scale, the resolved shear stresses on dislocation line depend on the grain orientation and can cause anisotropy in creep strain. Additional factors such as grain misorientations leading to asymmetrical dislocation pile-up and directional size effects, heterogeneous distribution of vacancies due to non-uniform emission/absorption by climbing dislocations and grain boundaries, can also influence the anisotropy in creep behaviour. Thus, resolving the creep mechanism at the polycrystalline length scale has significant potential in correlating the macroscopically observed secondary creep rates and creep rupture strain to microstructural features such as grain orientation, local misorientations, size, etc. 
Crystal plasticity (CP) constitutive models are adept in capturing this form of dependencies. However, a vast majority of the literature on $\mathrm{CP}$ is directed towards understanding elasto-viscoplastic behaviour and texture development in metallic alloys (Roters et al., 2010). In the last few decades CP-based finite element method (CPFEM) has been widely used not only to obtain equivalent macroscopic responses of representative volume elements (RVEs) (Chakraborty and Biner, 2016) but also discern damage localisation that may lead to eventual failure (Ghosh and Chakraborty, 2013). However, CP-based models of vacancy mediated creep is limited and has been ventured into recently by few authors.

Initial CP model for climb expressed climb rate through a power law equation on cubic slip systems in nickel-based superalloys (McHugh and Mohrmann, 1997). In (Fedelich, 1999), local climb at the $\gamma / \gamma^{\prime}$ phase boundary has been modelled as a recovery mechanism by approximating the resulting plastic deformation as shearing of the precipitates by dislocations on their initial slip plane. However, in both these models the influence of vacancy concentration on climb has not been considered. In the model proposed in Lebensohn et al. (2010), the ratio of the local vacancy concentration around the dislocation core to the equilibrium vacancy concentration has been considered to obtain the climbing shear stress. However, the model ignores the influence of vacancy diffusion rates on the climb rate. A more elaborate dislocation density-based model has been proposed in Geers et al. (2014) that considers vacancy concentration imbalances, vacancy diffusion rate, and balance of dislocation density evolution and vacancy emission/absorption at core of climbing dislocations, to obtain the effective shearing rate due to a combined glide-climb process. However, due to the presence of divergence equation for dislocation densities, the model is presently limited to artificial crystal structures with few slip systems.

In the present work, the climb model proposed in Geers et al. (2014) has been modified and is appended to the dislocation density-based glide model described in (Chakraborty and Biner, 2016) to model creep in single crystals of solution strengthened nickel-based alloys. The glide-climb model is elaborated in the next section. The calibration of the model parameters using the experimental data of alloy 617 (Wright et al., 2014) is described in Section 3. Subsequently, the response obtained from the model in polycrystals under constant strain rate and creep deformation is discussed in Section 4. The paper is concluded in Section 5.

\section{CP model of glide and climb}

In the CP model developed in this work, a multiplicative decomposition of the total deformation gradient $(\boldsymbol{F})$ into an elastic $\left(\boldsymbol{F}^{e}\right)$ and plastic $\left(\boldsymbol{F}^{p}\right)$ component is performed following

$$
\boldsymbol{F}=\boldsymbol{F}^{e} \boldsymbol{F}^{p}
$$

A virtual intermediate configuration is considered where the lattice shears along the different slip/climb systems without any stretching or rotation. The shearing of the lattice results in the plastic deformation gradient. Subsequently, the plastically deformed lattice is stretched and rotated to the current configuration via the elastic deformation gradient. In the intermediate configuration, the plastic velocity gradient $\left(\boldsymbol{L}^{p}\right)$ evolves as 


$$
\boldsymbol{L}^{p}=\dot{\boldsymbol{F}}^{p} \boldsymbol{F}^{p-1}=\sum_{\alpha=1}^{N s} \dot{\gamma}_{\text {glide }}^{\alpha} m_{0}^{\alpha} \otimes n_{0}^{\alpha}+\sum_{\alpha=1}^{N c} \dot{\gamma}_{\text {climb }}^{\alpha} m_{0}^{\alpha} \otimes m_{0}^{\alpha}
$$

where $\dot{\gamma}_{\text {glide }}^{\alpha}, \dot{\gamma}_{\text {climb }}^{\alpha}, m_{0}^{\alpha}, n_{0}^{\alpha} N s$ and $N c$, are the glide rate, climb rate, slip system direction, slip system normal, number of slip systems and number of climb systems, respectively. The glide rate is related to the velocity of the mobile dislocation density $\left(\rho_{M}^{\alpha}\right)$ following Orowan's equation

$$
\dot{\gamma}_{\text {glide }}^{\alpha}=\rho_{M}^{\alpha} b v^{\alpha}
$$

where the velocity of dislocation glide

$$
v^{\alpha}=\left\{\begin{array}{l}
l v \exp \left(\frac{-\Delta F_{G}}{K T}\left(1-\left(\frac{\left|\tau_{g}^{\alpha}\right|-g_{a}^{\alpha}}{g_{t}^{\alpha}}\right)^{p}\right)^{q}\right) \operatorname{sgn}\left(\tau_{g}^{\alpha}\right) \text { for }\left|\tau_{g}^{\alpha}\right|>g_{a}^{\alpha} \\
0 \quad \text { otherwise }
\end{array}\right.
$$

and $b, \rho_{M}^{\alpha}, l, v, \Delta F_{G}, K, T, \tau_{g}^{\alpha}, g_{a}^{\alpha}$ and $g_{t}^{\alpha}$ are the Burger's vector, mobile dislocation density, dislocation mean free path, jump frequency, activation enthalpy, Boltzman constant, temperature, resolved shear stress for glide, athermal and thermal resistances, respectively. The parameters $p$ and $q$ appearing in equation ( $3 \mathrm{~b})$ are fitting constants. The resolved shear stress for glide is obtained from the second Piola-Kirchhoff stress $(\boldsymbol{T})$ as

$$
\tau_{g}^{\alpha}=\boldsymbol{T}: m_{0}^{\alpha} \otimes n_{0}^{\alpha}
$$

The athermal resistance for the solution strengthened alloy is represented by

$$
g_{a}^{\alpha}=G b \sqrt{q_{\rho} \sum_{\xi} A^{\alpha \xi} \rho^{\xi}}
$$

and is due to the dislocation-dislocation interactions. In the above equation, $G, q_{\rho}$ and $A^{\alpha \xi}$ are the shear modulus, a constant parameter, and, components of self and latent hardening matrix, respectively. The thermal resistance to glide $\left(g_{t}^{\alpha}\right)$ is related to lattice frictional resistances and also due to solute drag from interstitials atoms. During the process of shearing, the mobile dislocations are trapped by parallel and cutting dislocations as well as solute atoms in solution strengthened alloys. In the absence of sufficient driving force to overcome these obstacles, dislocations can climb to bypass them. Dislocation climb is a thermally activated process by vacancy diffusion where the dislocation line moves in a direction perpendicular to the gliding plane. Depending on the local stress state, the equilibrium concentration of vacancies at the dislocation core increases or decreases than the surrounding matrix. This results in a net flux of vacancy to or away from the core causing a net upward or downward motion of the dislocation (Geers et al., 2014). This is represented by

$$
v_{c}^{\alpha}=-\frac{2 \pi D \alpha}{b \ln \left(r_{\infty}^{\alpha} / r_{c}\right)}\left[c_{e q}^{\alpha}-c_{0}\right]
$$


where $v_{c}^{\alpha}, D, b, c_{e q}^{\alpha}, c_{0}, r_{\infty}^{\alpha}$ and $r_{c}$ are the climb velocity, vacancy diffusion coefficient, Burger's vector, vacancy concentration at dislocation core and matrix, radial distance from the core where vacancy concentration is $c_{0}$ and, dislocation core radius, respectively. The equilibrium concentration of vacancy in the matrix at a given temperature depends on the vacancy formation energy $\left(Q_{v}\right)$ following

$$
c_{0}=\exp \left(-\frac{Q_{v}}{R T}\right)
$$

where $R$ is the gas constant. The radial distance from the dislocation core where the vacancy concentration can be assumed as dislocation free matrix vacancy concentration, can be represented by

$$
r_{\infty}^{\alpha}=\frac{1}{\sqrt{\rho^{\alpha}}}
$$

where $\rho^{\alpha}$ is the total dislocation density on any slip system. Hence, the effective diffusivity for climb between different climb systems can vary.

The equilibrium vacancy concentration at the dislocation core is modified due to the presence of osmotic force. Excess work needs to be performed to overcome this resistance which from the assumption of quasi-static climb motion of edge dislocations relates the climb stress, $\tau_{c}^{\alpha}$, to $c_{e q}^{\alpha}$ as

$$
c_{e q}^{\alpha}=c_{0} \exp \left(\frac{\tau_{c}^{\alpha} V_{m}}{R T}\right)
$$

where $V_{m}$ is the molar volume. The climb stress is represented as

$$
\tau_{c}^{\alpha}=\boldsymbol{T}: m_{0}^{\alpha} \otimes m_{0}^{\alpha}
$$

considering Peach-Koehler force on the climbing edge dislocation. The climb rate on individual system can subsequently be related to climb velocity following

$$
\dot{\gamma}_{\text {climb }}^{\alpha}=-\left(\rho_{M}^{\alpha}+\rho_{I}^{\alpha}\right) b v_{c}^{\alpha}=-\rho^{\alpha} b v_{c}^{\alpha} .
$$

where $\rho_{I}^{\alpha}$ and $\rho^{\alpha}$ are the immobile and total dislocation densities, respectively. The mobility of a dislocation is based on the resistance to glide. However, both immobile and mobile dislocations can climb under situations when the driving force is insufficient to activate gliding motion. As the dislocations absorb or emit vacancies to climb, the total concentration of vacancies evolve as

$$
\dot{c}_{0}=\sum_{\alpha} \dot{\gamma}_{\text {climb }}^{\alpha}-\nabla j
$$

where the first term is the contribution from vacancy emission or absorption from climbing dislocations while the second term is from the vacancy flux $j$ in the bulk with grain boundaries and free surfaces acting as source or sink. In the current model, the above equation is approximated as 


$$
\dot{c}_{0}=k \sum_{\alpha} \dot{\gamma}_{\mathrm{climb}}^{\alpha}
$$

since the gradient of bulk vacancy flux, which can significantly modify the bulk vacancy evolution rate, is not modelled explicitly in the current framework. However, the sole dependence of the bulk vacancy evolution rate on the vacancy absorption or emission rates from climbing dislocations can be an over simplification. Thus to incorporate the effect of gradient of bulk vacancy flux, a constant scaling factor, $k$, is introduced.

The shearing motion is associated with expansion, multiplication and annihilation of dislocations loops. Thus, a concurrent evolution of mobile and immobile dislocation density also occurs following

$$
\begin{aligned}
\dot{\rho}_{M}^{\alpha} & =\frac{k_{m u l}}{b} \sqrt{\sum_{\beta} \rho_{M}^{\beta}}\left|\dot{\gamma}_{\text {glide }}^{\alpha}\right|-\frac{2 R_{c \rho}}{b} \rho_{M}^{\alpha}\left(\left|\dot{\gamma}_{\text {glide }}^{\alpha}\right|+\left|\dot{\gamma}_{\text {climb }}^{\alpha}\right|\right) \\
& -\frac{\beta_{\rho} \sqrt{\rho^{\alpha}}}{b}\left(\left|\dot{\gamma}_{\text {glide }}^{\alpha}\right|+\left|\dot{\gamma}_{\text {climb }}^{\alpha}\right|\right)+C_{M}\left|\dot{\gamma}_{\text {climb }}^{\alpha}\right|
\end{aligned}
$$

and

$$
\dot{\rho}_{I}^{\alpha}=\frac{\beta_{\rho} \sqrt{\rho^{\alpha}}}{b}\left(\left|\dot{\gamma}_{\text {glide }}^{\alpha}\right|+\left|\dot{\gamma}_{\text {climb }}^{\alpha}\right|\right)-C_{M}\left|\dot{\gamma}_{\text {climb }}^{\alpha}\right|
$$

where the first term in equation (14) is the dislocation multiplication, second term is the self annihilation, third term is the dislocation trapping and fourth term is the mobilisation due to climb. As the dislocations are trapped, the mobile dislocation density decreases with a subsequent increase in immobile dislocation density, as shown in equation (15). Similarly, climb increases the mobile dislocation density via bypassing the obstacles. In equations (14) and (15), $k_{m u l}, R_{c \rho}, \beta_{\rho}$ and $C_{M}$ are phenomenological scaling parameters calibrated from experiments. These parameters can be temperature dependent, however, assumed athermal in this work.

\subsection{Numerical implementation}

The equations governing the evolution of internal state shown above [equations (1) to (11) and (13) to (15)] are solved concurrently with the quasi-static stress equilibrium, to obtain the time dependent deformation of the body. Finite element method (FEM) is used to evaluate the temporally and spatially varying velocity field where the constitutive equations are integrated using the stress-update algorithm described in Balasubramanian (1998). The model is implemented in multi-physics object oriented simulation environment (MOOSE), which is an open source finite element tool to solve partial differential equations (Gaston et al., 2009). The tool is based on $\mathrm{C}++$ that allows easy code development. This tool utilises the iterative Jacobian free Newton Krylov (JFNK) method for nonlinear problems. The use of iterative Krylov subspace method enables good scalability of large simulations in parallel environment. Furthermore, the use of JFNK method provides quadratic convergence without the incorporation of the exact tangent modulus. 


\section{Determining model parameters using constant strain rate and creep experiments}

The experimental data for alloy 617 provided in Wright et al. (2014) is used to calibrate the model parameters. The glide parameters are calibrated using the stress-strain response of the uniaxial constant strain rate experiment at room temperature. Since, the equilibrium vacancy concentration and diffusivity are extremely low at room temperature, plastic deformation only occurs by dislocation glide. Thus, only the glide parameters can be calibrated from this experimental data.

Figure 1 (a) Pole figure of [001] direction along loading axis (b) Euler angle, $\phi$, in degrees, in the synthetic microstructure (see online version for colours)

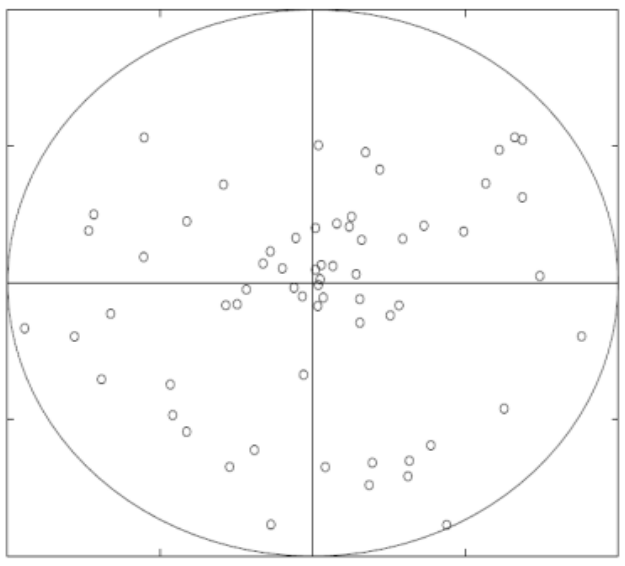

(a)

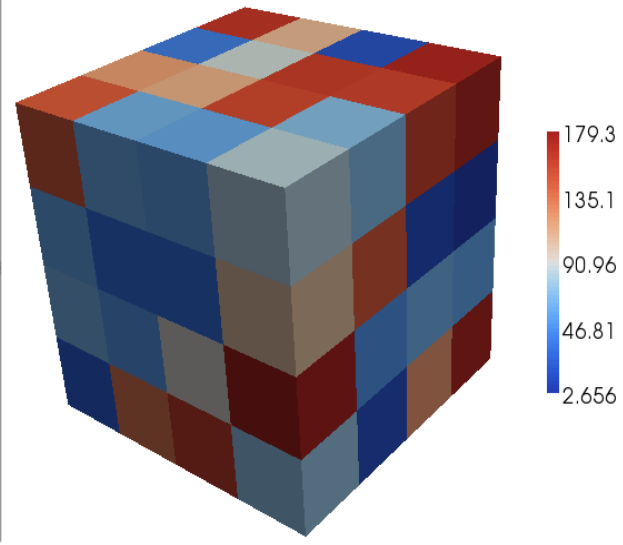

(b)

For the calibration, a microstructure of size $200 \times 200 \times 200 \mu \mathrm{m}^{3}$ with 64 cubic grains is considered similar to Chakraborty and Biner (2016). Random Euler angles are assigned to each grain and the pole figure of $\left[\begin{array}{lll}0 & 0 & 1\end{array}\right]$ axis along the loading direction is shown in Figure 1(a). The microstructure is discretised with 64 hexahedral elements, where, one element is associated with every grain. The discretisation strategy is relatively accurate to obtain average plastic properties of strain hardening material (Chakraborty and Biner, 2016), as is the case here. The distribution of Euler angle, $\phi$, for the synthetic microstructure utilised in this work is shown in Figure 1(b).

In the CPFEM simulations, symmetry boundary condition is used and one of the faces is uniaxially displaced with a constant strain rate of $10^{-3} / \mathrm{s}$. The initial density of mobile and immobile dislocations on every slip system is chosen as $1 / \mu \mathrm{m}^{2}$, corresponding to well annealed face centred cubic material. The material parameters are estimated through an iterative process where the difference between the experimental and simulated engineering stress strain curve till 5\% strain is used as the criterion to terminate the process. The initial guess of parameter values to start the iterations is chosen from (Chakraborty and Biner, 2016). A comparison of the engineering stress-strain curve is shown in Figure 2 and the corresponding set of parameters is tabulated in Table 1. $A^{\alpha \xi}$ is taken as 1 for self hardening and 0.4 for latent hardening. 
Figure 2 Comparison of experimental and simulated engineering stress-strain curve at room temperature

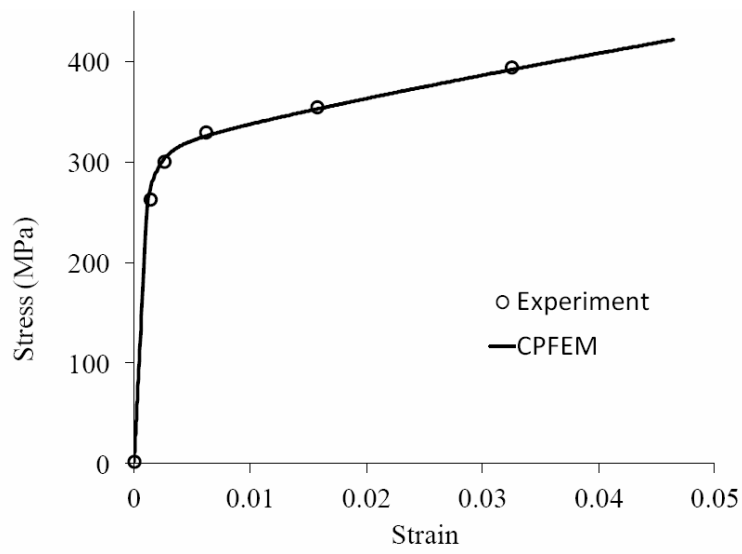

Source: Wright et al. (2014)

Figure 3 (a) Material line in the microstructure (b) Representative grains (c) Euler angle, variation (see online version for colours)

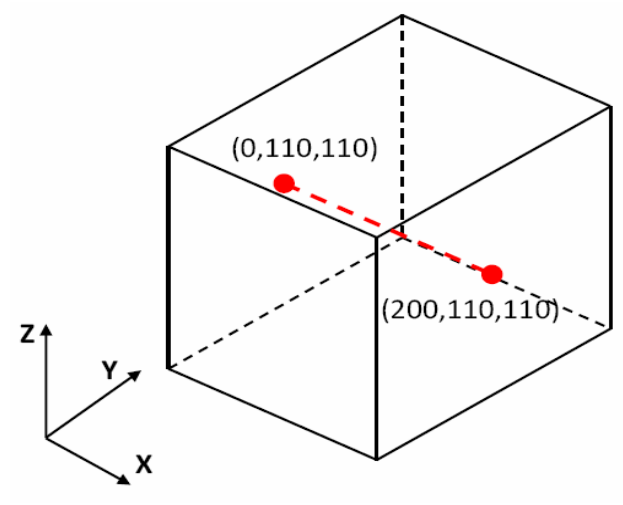

(a)

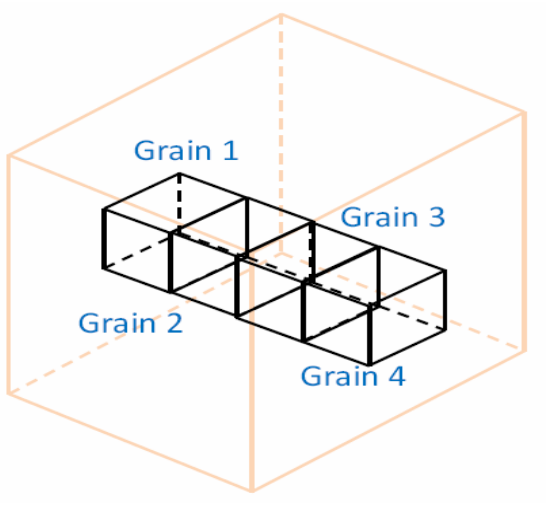

(b)

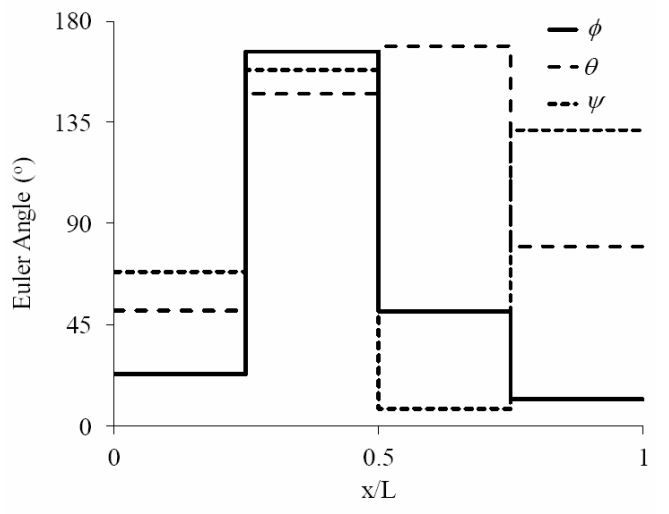

(c) 
Figure 4 Variation of (a) normal stress component along loading direction and (b) average dislocation density of all slip systems, in the grains along the material line at $\sim 0.05$ macroscopic strain

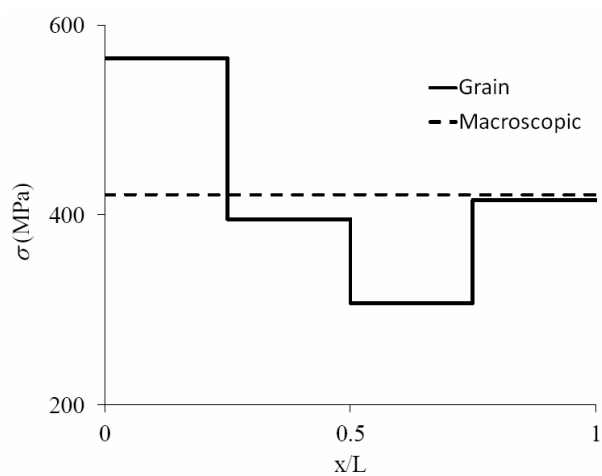

(a)

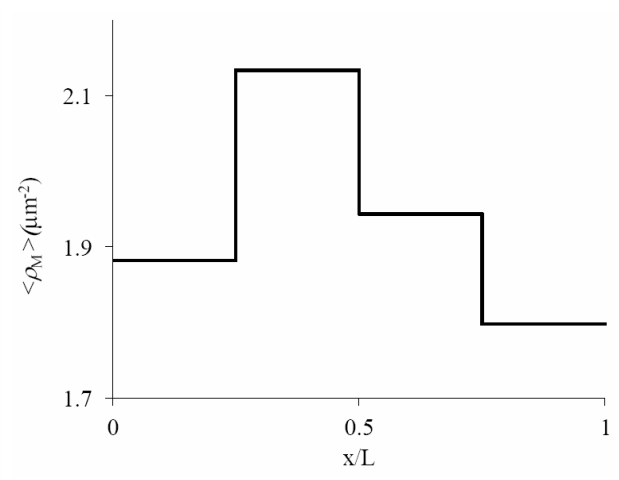

(b)

Figure 5 Variation of RMS value of Schmid factor

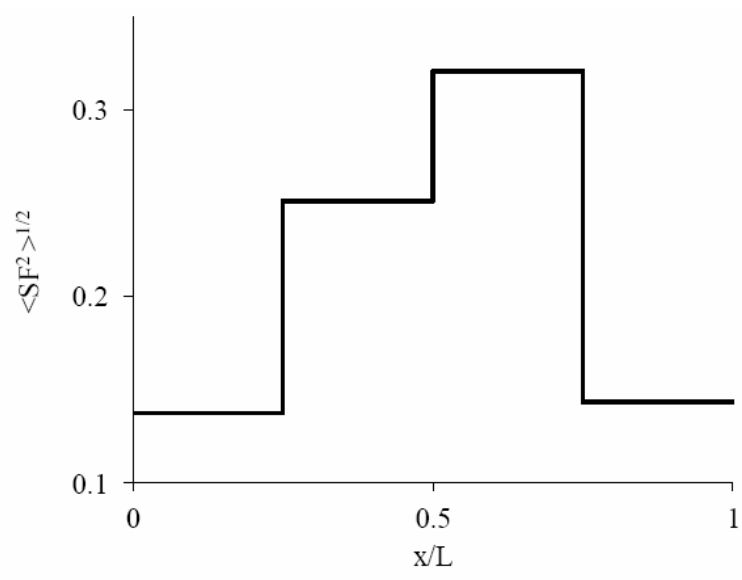

Depending on the orientation of grains, the evolution of stress, strain and internal state variables in the microstructure can vary significantly. To demonstrate this heterogeneous evolution of variables, a material line in the microstructure is chosen as shown in Figures 3(a) and (b). The Euler angles of the grains on this material line are shown in Figure 3(c). In Figure 4, the evolution of stress component along the loading direction [Figure 4(a)], and, the average mobile dislocation density [Figure 4(b)], in each grain on the material line at approximately 5\% macroscopic strain, is shown. A higher average mobile dislocation density in one grain as compared to another signifies an increased slip activity in the former. Since, the extent of stress relaxation is directly related to slip activity, hence, the grains with higher average mobile dislocation density shows lower stresses. A one-one correlation cannot be observed in this case, since the multiplication of mobile density on a slip system also considers the density on other slip systems. The root mean squared (RMS) value of the Schmid factor (SF) provides a much better description of the relaxed stress state in this case and is shown in Figure 5. Since, the SF provides a 
direct measure of resolved shear stress, which governs the slip rate on a slip system; hence, the corresponding RMS value provides a satisfactory representation of grain scale plasticity and stress relaxation.

Figure 6 Comparison of temporal evolution of (a) stress and (b) mobile dislocation density in the grains with macroscopic applied strain

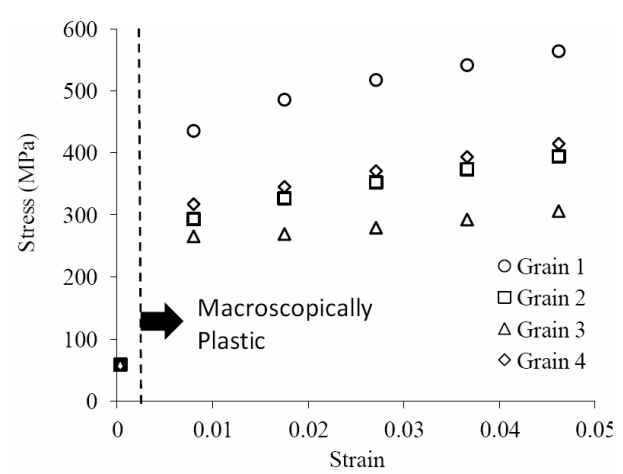

(a)

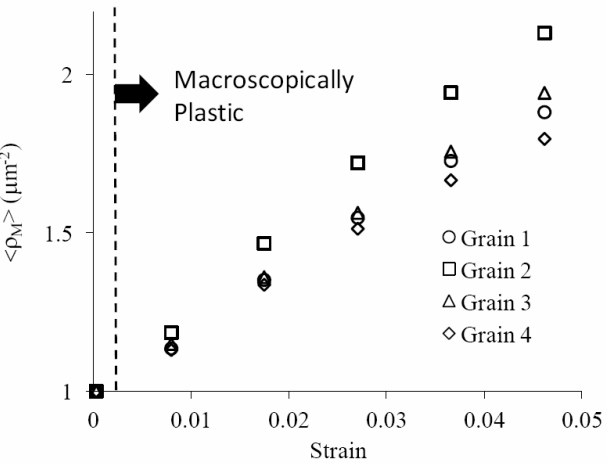

(b)

The evolution of stress component along the loading direction and average mobile dislocation density with the applied strain in the four grains on the material line is compared in Figure 6. As can be observed from the figure, in the elastic regime the stresses are same owing to the isotropic elastic properties considered here. However, the plastic behaviour shows strong anisotropy with grain 1 and grain 3 having the maximum and minimum local yield strength values, respectively. A difference of approximately $200 \mathrm{MPa}$ can be observed in this case. Furthermore, the grains also show different hardening behaviour with the largest difference observable between grains 1 and 3 .

The calibration of uniaxial strain rate data provides parameter values only for the glide model. Under the conditions of strain rate utilised in this test $\left(10^{-3} / \mathrm{s}\right)$, plastic deformation due to climb is negligible and hence the parameters related to climb cannot be evaluated from this test. Thus, to obtain the climb related parameters, the unaxial creep strain-time response at $750^{\circ} \mathrm{C}$ and tensile stress of $135 \mathrm{MPa}$ for the alloy 617 provided in Wright et al. (2014) is utilised. The same representative volume shown above is considered for the uniaxial creep simulation. The equilibrium vacancy concentration is obtained from equation (7) as $4 \times 10^{-8}$ where $Q_{v}=1.5 \mathrm{eV}$ is the vacancy formation energy (Johnson, 1966). The other parameters such as $k, C_{M}$ and $D$, shown in the above equations, are obtained from an iterative technique where the parameters have been varied till the creep strain-time responses in the secondary creep regime between experiment and simulation are comparable. The parameter values obtained from calibration is tabulated in Table 1. A comparison of the bulk diffusivity parameter, $D$, evaluated from the calibration and that obtained from the empirical (Rothova et al., 2010) equation

$$
D=9.2 \times 10^{-5} \exp \left(-\frac{Q_{m v}}{R T}\right)+3.7 \times 10^{-3} \exp \left(-\frac{Q_{d v}}{R T}\right)\left(m^{2} / \mathrm{s}\right)
$$


reveals that this calibrated parameter value is six orders of magnitude higher than what obtained in experiments $\left(6 \times 10^{-19} \mathrm{~m}^{2} / \mathrm{s}\right)$. In the above equation, values of $278 \mathrm{~kJ} / \mathrm{mol}$ and $357 \mathrm{~kJ} / \mathrm{mol}$ are used for the mono $\left(Q_{m v}\right)$ and divancanies $\left(Q_{d v}\right)$ molar activation energies, respectively (Rothova et al., 2010). Similar scaled up values of diffusivity has been used in Geers et al. (2014) where the bypassing of dislocation around circular precipitates by climb has been investigated.

Figure 7 Comparison of experimental and simulated creep strain - time curve at $750 \mathrm{oc}$ and $135 \mathrm{MPa}$

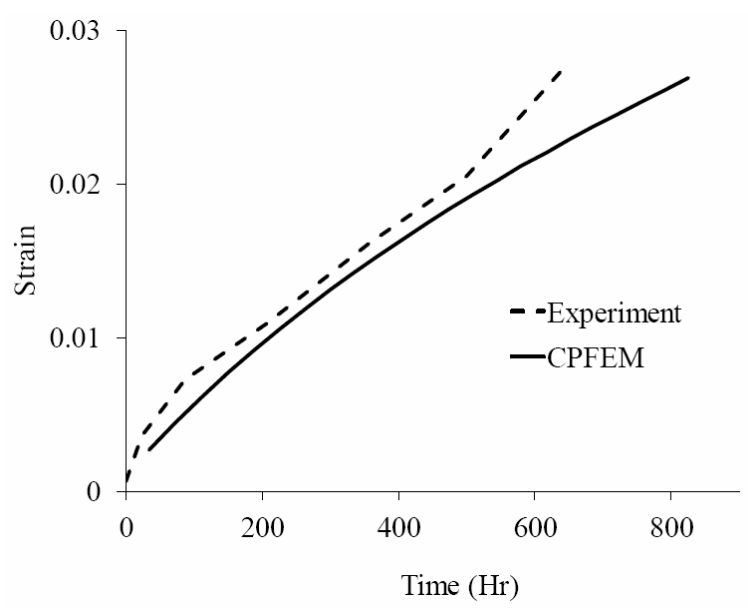

Source: Wright et al. (2014)

Figure 8 (a) Creep strain and (b) RMS value of absolute glide and climb rates, in grains along material line shown in Figure 4, at time 825 hrs

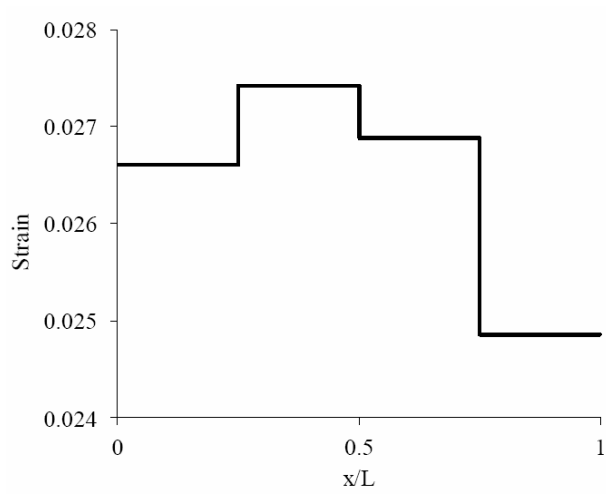

(a)

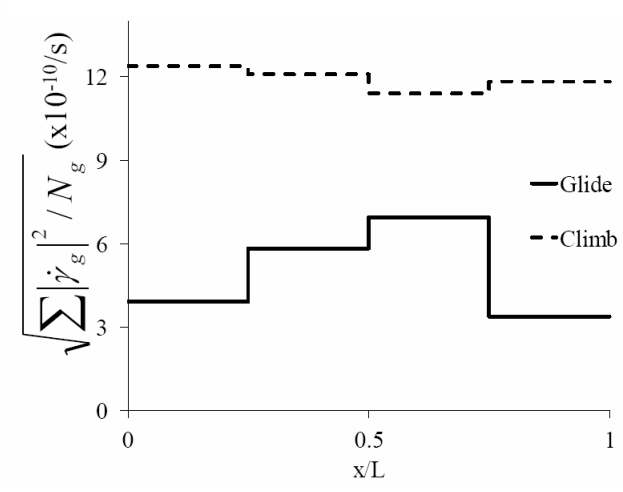

(b)

A comparison of creep strain-time responses between CPFEM simulation and experiment is shown in Figure 7. For the calibrated set of parameters, a reasonable agreement with the experimental curve can be observed from the figure. The component of creep strain tensor along the loading direction in the grains on the material line shown in Figure 4 at 825 hrs is shown in Figure 8(a). As can be observed from the figure, the effect of crystal 
orientation does affect the creep strain in the individual grains, though not as conspicuous as under constant strain rate experiments. The reason can be easily explained from Figure 8(b) where the RMS values of glide and climb rates have been compared. Though the RMS value of the glide rate is approximately four times lower than the climb rate, it is much more anisotropic, as can be observed from the variations. Since, the shear due to climb contributes to a greater degree to the creep strain, thus the overall orientation dependence of creep strain is reduced. The evolution of creep strain rate with time in the individual grains along the material line is shown in Figure 9. Apart from grain 4, all the others have similar creep strain rates.

Figure 9 Evolution of creep strain with time in grains along the material line shown in Figure 4

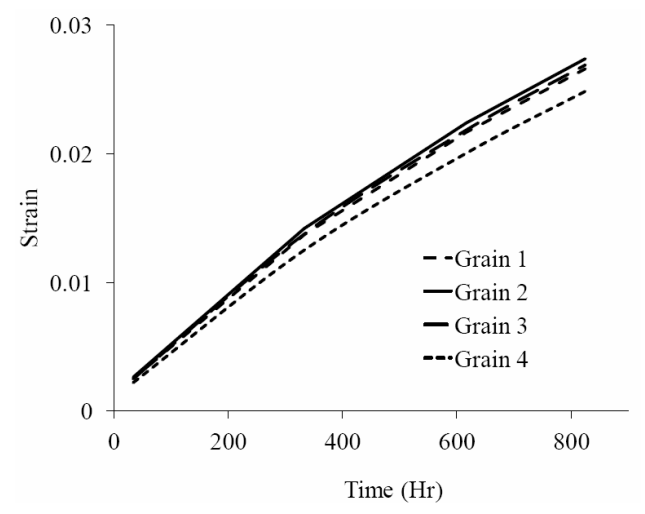

Table 1 Calibrated parameters from constant strain rate and creep simulations at room temperature and $750^{\circ} \mathrm{C}$, respectively

\begin{tabular}{lccccc}
\hline $1(\mathrm{~nm})$ & 1.5 & $\mathrm{~b}(\mathrm{~nm})$ & 0.248 & $\beta_{\rho}$ & 0.018 \\
$\mathrm{v}(\mathrm{s})$ & $10^{10}$ & $D\left(\mu \mathrm{m}^{2} / \mathrm{s}\right)$ & 0.2 & $C_{M}$ & 1 \\
$\Delta F_{g}(J)$ & $8 \times 10^{-7}$ & $r_{c}$ & $4 \mathrm{~b}$ & $q_{\rho}$ & 1 \\
$g_{t}^{\alpha}(\mathrm{MPa})$ & 125 & $V_{m}\left(\mathrm{~cm}^{3} / \mathrm{mol}\right)$ & 6.58 & $R_{c \rho}(\mathrm{nm})$ & 1 \\
$p$ & 0.3 & $k$ & $10-7$ & $G(\mathrm{GPa})$ & 80 \\
$q$ & 1.4 & $k_{m u l}$ & 0.018 & & \\
\hline
\end{tabular}

\section{Verification of the model parameters}

The model parameters have been verified by comparing the creep strain evolution at $850^{\circ} \mathrm{C}$ and $950^{\circ} \mathrm{C}$ with experimental data. The equilibrium vacancy concentration in the matrix is obtained from equation (18). These are $2 \times 10^{-7}$ and $7 \times 10^{-7}$ at temperatures of $850^{\circ} \mathrm{C}$ and $950^{\circ} \mathrm{C}$, respectively. The diffusivity at the different temperatures is obtained from equation (17) and scaled with the factor used in calibration. At $850^{\circ} \mathrm{C}$ the modified diffusivity is thus $4 \mu \mathrm{m} / \mathrm{s}^{2}$.

Prior to validating the creep responses, constant strain rate simulations of the same synthetic microstructure are performed to investigate the effect of temperature on the yield stress and strain hardening behaviour. The comparison is shown in Figure 10(a). As 
can be observed from the figure, a sharp decrease in yield stress occurs from room temperature to $750^{\circ} \mathrm{C}$. This reduction solely happens due to the presence of temperature term in equation (3b). Thermally dependent glide resistant parameters have not been varied in this case. The strain hardening behaviour however remains almost the same since dislocation multiplication, trapping, annihilation and recovery parameters do not vary with temperature. A comparison of the $0.2 \%$ offset yield strength variation with temperature between CPFEM and experiment (special metals) is shown in Figure 10(b). A reasonable agreement can be observed from the figure.

Figure 10 Comparison of temperature varying, (a) engineering stress-strain response from CPFEM simulations (b) $0.2 \%$ offset yield strength (special metals)

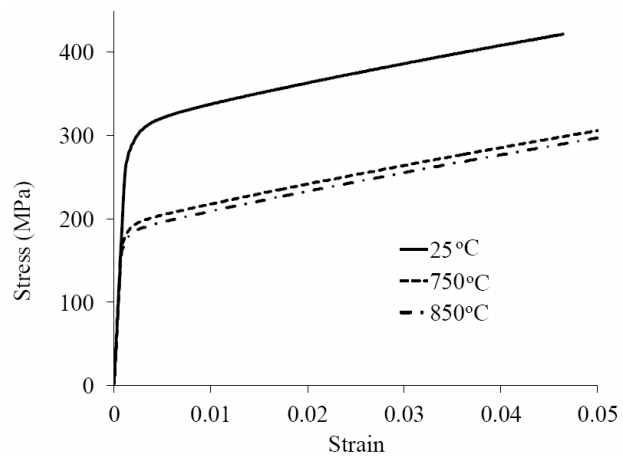

(a)

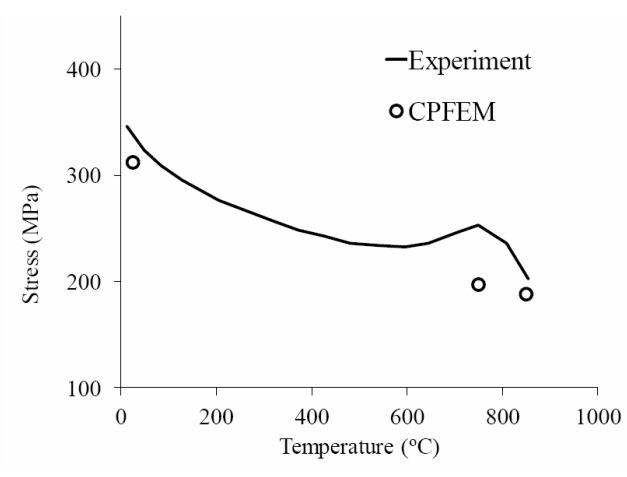

(b)

Figure 11 A comparison of normalised loading direction stress component distribution along a material line at $\sim 5 \%$ strain between two different temperature

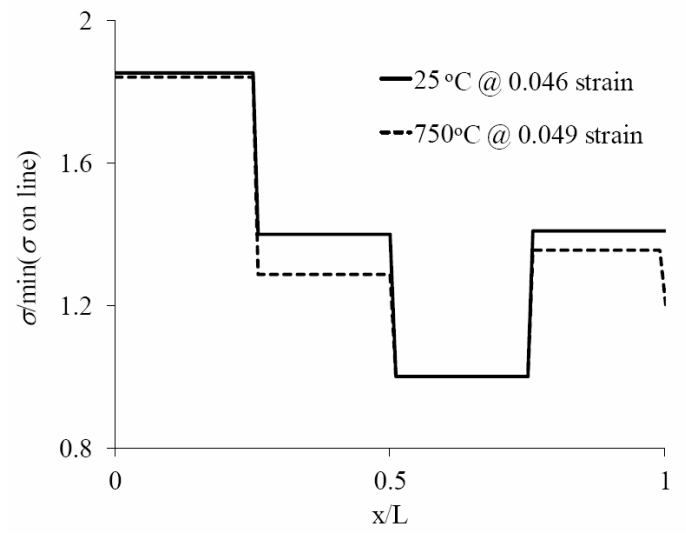

The influence of temperature on the microstructural stress distribution is investigated by comparing the stress component along the loading direction in grains along a material line (Figure 4) and is shown in Figure 11. The stress values are normalised with the minimum stress on the line. Though in two of the grains the normalised stress values are the same, signifying a constant shift in the stress response, there are observable differences in the other two grains and can be due to a difference in relative strain hardening rate. Subsequently, the model is further verified by comparing the secondary 
creep strain evolutions between CPFEM and experiments at $850^{\circ} \mathrm{C}$, and, applied uniaxial stresses of 63 and $54 \mathrm{MPa}$. In addition to the scaled diffusivities as discussed above, the parameter, $k$, affecting the vacancy evolution rate is also modified to $10^{-9}$. The rational for the second modification is that with increase in temperature both vacancy generation and mobility becomes higher, which can lower the effective vacancy addition/removal rate from the matrix. The comparisons are shown in Figure 12. While the calibrated model parameters satisfactorily reproduce the secondary creep strain evolution at $63 \mathrm{MPa}$, a noticeable difference can be observed for $54 \mathrm{MPa}$. This signifies that the stress dependence of the climb process is possibly much stronger than obtained by balancing the Peach-Koehler and osmotic forces at the dislocation core.

Figure 12 Comparison of creep strain response between CPFEM and experiments (Wright et al., 2014) at a temperature of $850^{\circ} \mathrm{C}$ and applied stress levels of (a) 63 and (b) $54 \mathrm{MPa}$

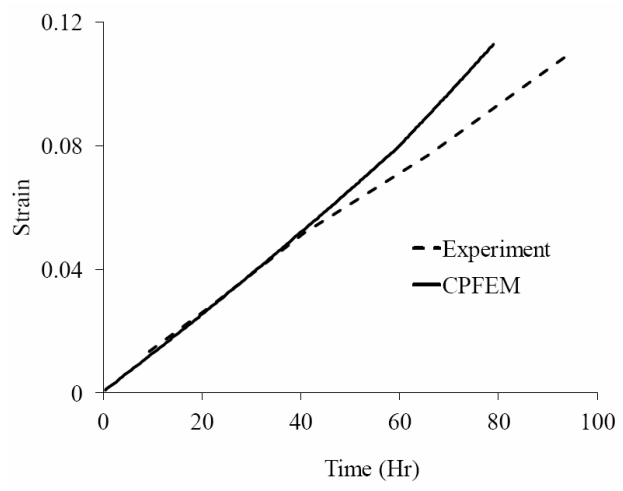

(a)

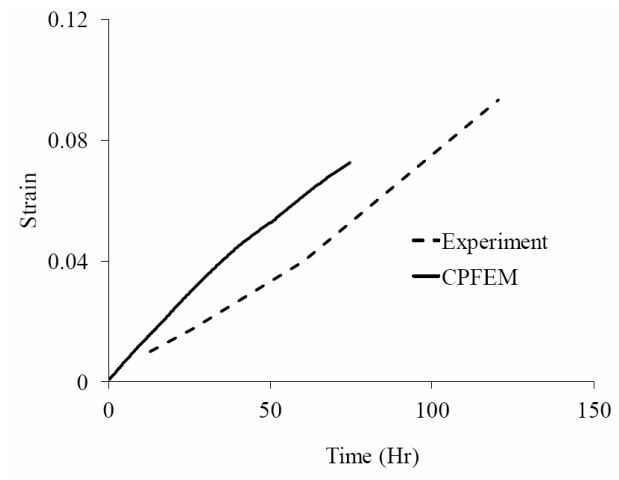

(b)

Figure 13 Comparison of RMS values of climb rates between loading stress levels of 63 and 54 $\mathrm{MPa}$, and, temperature of $850^{\circ} \mathrm{C}$ at (a) 75 hours and (b) $\sim 7 \%$ macroscopic strain, along the material line shown in Figure 4

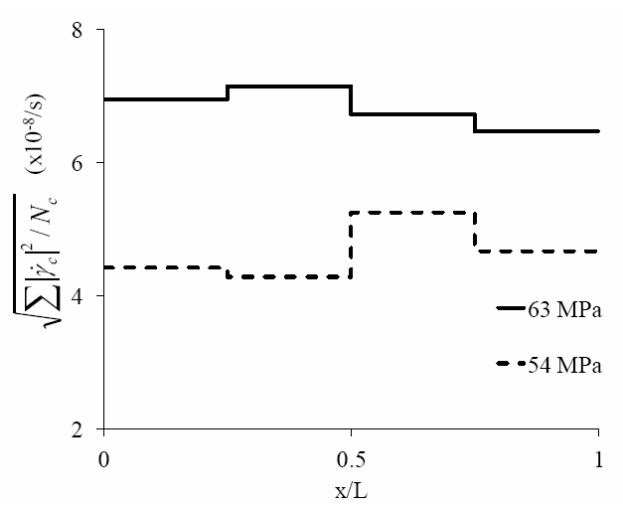

(a)

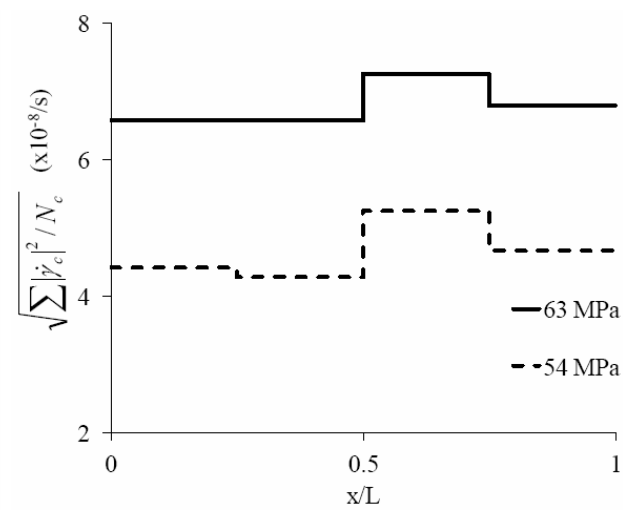

(b)

The RMS value of the climb rates along the material line (Figure 4) at 75 hours for these two cases is also compared in Figure 13(a). As expected, the RMS value of climb rate at 
applied stress level of $63 \mathrm{MPa}$ is higher than that at $54 \mathrm{MPa}$. However, the spatial distributions of the stress values are different, which can be attributed to the disparity in the dislocation densities that can alter the climb rates. Though the compared states in Figure 13(a) are at the same time, the macroscopic strain levels are different, viz. $~ 7 \%$ and $\sim 11 \%$ under 54 and $63 \mathrm{MPa}$, respectively. With the strain levels differing by a factor of 1.5, the microscopic dislocation densities can be significantly dissimilar and can considerably alter the climb rates. It is expected that at similar strain levels, microscopic climb rates or atleast their spatial distribution can be similar. To explore this fact, the RMS values of the climb rates are compared at the same macroscopic strain level and shown in Figure 13(b). In this figure it can be seen that though the RMS value of the climb rates is higher for $63 \mathrm{MPa}$, the spatial distributions are the same. The creep strain response at $950^{\circ} \mathrm{C}$ and $28 \mathrm{MPa}$ is further compared in Figure 14. The scaled up diffusivity of 46 at this condition gives a significantly higher climb rate than observed in experiments. However, when the diffusivity is unaltered (same as $850^{\circ} \mathrm{C}$ ), a reasonable comparison with the experimental response can be obtained (Figure 14).

Figure 14 Creep strain evolution at $950^{\circ} \mathrm{C}$ and $28 \mathrm{MPa}$

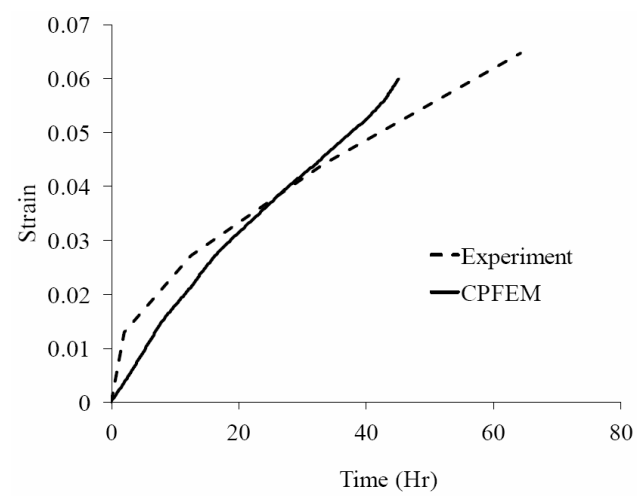

Source: Wright et al. (2014)

\section{Conclusions}

Polycrystalline scale models of viscoplasticity and creep can provide the necessary resolution to capture the effect of grain size, orientation, grain boundary character, misorientation, etc. on the macroscopic creep behaviour. Based on this motivation a CP model has been developed in the present work wherein the mechanisms of dislocation glide and climb are coupled to obtain a microscopic description of the empirical power-law creep. While the glide rate on each slip system has been represented by the well known mechanical threshold stress (MTS) model, the climb rate on every climb system has been denoted by vacancy absorption/emission at dislocation core. By utilising this model, the uniaxial constant strain rate and creep response of alloy 617 has been investigated. From a systematic calibration and validation study, the model has been shown to capture the responses reasonable well. However, the scaling of diffusivity parameter has been necessary to obtain satisfactory comparison. Though the diffusivity parameter in the model is related to the bulk diffusivity, a difference of six orders between the two necessitates a closer look into the effects that can cause such a 
difference. Furthermore, a coupling of the bulk vacancy evolution partial differential equation to the stress divergence is necessary to obtain more realistic predictions.

\section{References}

Balasubramanian, S. (1998) Polycrystalline Plasticity: Application to Deformation Processing of Light Weight Metals, PhD thesis, Massachusetts Institute of Technology.

Chakraborty, P. and Biner, S.B. (2016) 'Crystal plasticity modeling of irradiation effects on flow stress in pure-iron and iron-copper alloys', Mechanics of Materials, Vol. 101, pp.71-80.

Chomette, S.J., Gentzbittel, M. and Viguier, B. (2010) 'Creep behaviour of as received, aged and cold worked INCONEL 617 at $850 \mathrm{oC}$ and 950oC', Journal of Nuclear Materials, Vol. 399, Nos. 2-3, pp.266-274.

Fedelich, B. (1999) 'A microstructure-based constitutive model for the mechanical behaviour at high temperatures of nickel-base single crystal superalloys', Computational Materials Science, Vol. 16, Nos. 1-4, pp.248-258.

Gaston, D., Newman, C., Hansen, G. and Lebrun-Grandie, D. (2009) 'MOOSE: a parallel computational framework for coupled systems of nonlinear equations', Nuclear Engineering and Design, Vol. 239, No. 10, pp.1768-778.

Geers, M.G.D., Cottura, M., Appolaire, B., Busso, E.P., Forest, S. and Villani, A. (2014) 'Coupled glide-climb enhanced crystal plasticity', Journal of the Mechanics and Physics of Solids, Vol. 70, pp.136-153.

Ghosh, S. and Chakraborty, P. (2013) 'Microstructure and load sensitive fatigue crack nucleation in Ti-6242 using accelerated crystal plasticity FEM simulations', International Journal of Fatigue, Vol. 48, pp.231-246.

Giglio, M., Fossati, M., Lumassi, D. and Manes, A. (2013) 'Use of numerical simulations in damage assessment due to high velocity impact', International Journal of Materials and Structural Integrity, Vol. 7, No. 4, pp.215-231.

Johnson, R.A. (1966) 'Point-defect calculations for an FCC lattice', Physical Review, Vol. 145, No. 2, pp.423-433.

Lebensohn, R.A., Hartley, C.S., Tome, C.N. and Castelnau, O. (2010) 'Modeling the mechanical response of polycrystals deforming by climb and glide', Philosophical Magazine, Vol. 90, No. 5, pp.567-583.

Lillo, T.M. and Wright, R.N. (2015) 'Microstructural characterization of alloy 617 crept into the tertiary regime', Proceedings of the 2015 ASME Pressure Vessels and Piping Conference, Boston, Massachusetts, 19-23 July.

McHugh, P.E. and Mohrmann, R. (1997) 'Modelling of creep in a Ni base superalloy using a single crystal plasticity model', Computational Materials Science, Vol. 9, Nos. 1-2, pp.134-140.

Roters, F., Eisenlohr, P., Hantcherli, L., Tjahjanto, D., Bieler, T.R. and Raabe, D. (2010) 'Overview of constitutive laws, kinematics, homogenization and multi-scale methods in crystal plasticity finite-element modeling: theory, experiments, applications', Acta Materialia, Vol. 58, No. 4, pp.1152-1211.

Rothova, V., Svoboda, M. and Bursik, J. (2010) 'Grain boundary self-diffusion in nickel: comparison of theoretical and experimental diffusivities', Metal 2010-International Conference on Metallurgy and Materials.

Special Metals [online] http://www.specialmetals.com/assets/smc/documents/alloys/inconel/ inconel-alloy-617.pdf (accessed July 2018).

Swindeman, R.W. and Swindeman, M.J. (2008) 'A comparison of creep models for nickel base alloys for advanced energy systems', International Journal of Pressure Vessels and Piping, Vol. 85, Nos. 1-2, pp.72-79.

Wright, J.K., Carroll, L.J. and Wright, R.N. (2014) Creep and Creep-Fatigue of Alloy 617 Weldments, INL/EXT-14-32966. 